\title{
A SIMPLE CONSTRUCTION OF STEIN'S COMPLEMENTARY SERIES REPRESENTATIONS
}

\author{
SIDDHARTHA SAHI \\ (Communicated by Jonathan M. Rosenberg)
}

\begin{abstract}
We given an elementary construction of Stein's complementary series for $\mathrm{GL}(2 n)$ over an arbitrary local field $\mathbf{F}$, and determine their restrictions to the "mirabolic" subgroup $P_{2 n} \approx \mathrm{GL}(2 n-1, \mathbf{F}) \ltimes \mathrm{F}^{2 n-1}$. Taken together with the results in [S], this allows one to calculate the adduced representation $A \pi$ for an arbitrary irreducible, unitary representation $\pi$ of $G L(n, \mathbb{C})$.
\end{abstract}

The purpose of this paper is (1) to give an elementary construction of Stein's complementary series representations [St] for $\mathrm{GL}(2 n, \mathbb{F})$, and (2) to explicitly identify their restriction to the "mirabolic" subgroup $P_{2 n} \approx \mathrm{GL}(2 n-1, \mathbb{F}) \ltimes$ $\mathbb{F}^{2 n-1}$.

The main point is that the Bernstein-Zelevinsky theory of the (highest) derivative $[B]$ gives an answer to (2) for nonarchimedean $\mathbb{F}$ (where analogous representations were constructed by Godement); namely that the restriction corresponds to the Stein (Godement) representation for $\operatorname{GL}(2 n-2, \mathbb{F})$ with the same parameter.

For archimedean fields it is not known (at least to the author) how to extend the above arguments to answer (2). However, it is reasonable to expect that the answer should be the same for all fields.

Having "guessed" the answer to (2), it seems clear that one should try an inductive argument (on $n$ ) to prove it. Such an argument is given in $\S 2$ and leads to the construction referred to in (1) above. The interesting feature of this argument is that it uses almost nothing from $[\mathrm{B}]$ or $[\mathrm{St}]$, and reduces the problem to the representation theory of $\mathrm{GL}(2, \mathrm{~F})$ where the corresponding facts are well known (and easy to prove).

\section{STATEMENT OF THE MAIN THEOREM}

1.1. Let $\mathbb{F}$ be a local field. We begin by describing the degenerate series studied by Stein and Godement.

Received by the editors October 17, 1988 and, in revised form, February 20, 1989.

1980 Mathematics Subject Classification (1985 Revision). Primary 22E46, 22E45, 22 E50.

Key words and phrases. Stein's complementary series, adduced representation.

Research supported in part by NSF Grants DMS-812079-05 and 215-6190. 
Let us write $G_{2 n}$ for $\mathrm{GL}(2 n, \mathbb{F})$, and write a typical element $g \in G_{2 n}$ as

$$
g=\left[\begin{array}{ll}
a & c \\
b & d
\end{array}\right] \quad a, b, c, d \in M_{n}(\mathbb{F})
$$

where $\operatorname{det}(g) \neq 0$, and $M_{n}(\mathbb{F})$ is the space of $n \times n$ F-matrices.

Also, let us write

$$
Q_{n}=\left\{\left[\begin{array}{ll}
a & c \\
0 & d
\end{array}\right]\right\}, \quad S_{n}=\left\{\left[\begin{array}{ll}
a & c \\
0 & 1
\end{array}\right]\right\}, \quad N_{n}=\left\{\left[\begin{array}{ll}
1 & c \\
0 & 1
\end{array}\right]\right\}
$$

and $\bar{Q}_{n}=\left\{\left[\begin{array}{ll}a & 0 \\ b & d\end{array}\right]\right\}$ for the indicated subgroups of $G_{2 n}$. Notice that $N_{n}$ can be identified with the additive group of $M_{n}(\mathbb{F})$.

Let us fix a Haar measure on $\mathbb{F}$, and use it to define $d \mu$ on $M_{n}(\mathbb{F})$. Also let $\mid$ be the absolute value on $\mathrm{F}$ (see Weil [W]). For $a$ in $M_{n}(\mathbb{F})$ we shall write $|a|$ as shorthand for $|\operatorname{det} a|$.

Consider the one-dimensional character $L_{s}$ of $\bar{Q}_{n}$ given by

$$
L_{s}\left(\left[\begin{array}{ll}
a & 0 \\
b & d
\end{array}\right]\right)=|a|^{-s}|d|^{s} \quad \text { for } s \in \mathbb{C} .
$$

When $s$ is imaginary, $L_{s}$ is unitary, and we may consider the unitarily induced representation

$$
\sigma_{n}^{s}=\sigma^{s}=\operatorname{Ind} \bar{Q}_{n}^{G_{2 n}}\left(L_{s}\right) \text {. }
$$

Using the Gelfand-Naimark decomposition, $\sigma^{s}$ may be realized explicitly on

$$
L^{2}\left(N_{n}\right) \approx L^{2}\left(M_{n}(\mathbb{F})\right)
$$

as follows:

$$
\sigma^{s}(g) f(x)=\left|a^{\prime}\right|^{-(n / 2+s)}\left|d^{\prime}\right|^{n / 2+s} f\left(x^{\prime}\right)
$$

where $a^{\prime}, d^{\prime}, x^{\prime}$ are obtained by solving

$$
\left[\begin{array}{ll}
1 & x \\
0 & 1
\end{array}\right]\left[\begin{array}{ll}
a & c \\
b & d
\end{array}\right]=\left[\begin{array}{cc}
a^{\prime} & 0 \\
b^{\prime} & d^{\prime}
\end{array}\right]\left[\begin{array}{cc}
1 & x^{\prime} \\
0 & 1
\end{array}\right]
$$

This gives

$$
a^{\prime}=(a+x b), \quad x^{\prime}=(a+x b)^{-1}(c+x d)
$$

and

$$
\left|d^{\prime}\right|=|g|\left|a^{\prime}\right|^{-1}=|g||a+x b|^{-1} .
$$

It is a classical result of Gelfand and Naimark that the representations $\left\{\sigma^{s}: s \in i \mathbb{R}\right\}$ are irreducible and unitary as representations of $G_{2 n}$. In fact, the representations stay irreducible upon restriction to $Q_{n}$, or even $S_{n}$ (see $[\mathrm{S}])$.

Following Stein [St], we consider the (unitary) operators $B(s)$ on $L^{2}\left(M_{n}\right)$ given by

$$
B(s) f(x)=|x|^{s} f(x), \quad \text { for } s \in i \mathbb{R}
$$


and their Fourier transforms

$$
A(s)=\mathscr{F}^{-1} B(s) \mathscr{F}, \quad \text { for } s \in i \mathbb{R} .
$$

The operators $A(s)$ may be used to define the normalized series $\mathscr{N}_{n}^{s}=\mathscr{N}^{s}$ for $s \in i \mathbb{R}$

$$
\mathscr{N}^{s}(g)=A(-s) \sigma^{s}(g) A(s) .
$$

The representations $\mathscr{N}^{s}(g)$ are unitarily equivalent to $\sigma^{s}(g)$ and satisfy

$$
\mathscr{N}^{s}(q)=\mathscr{N}^{0}(q)=\sigma^{0}(q)
$$

for all $s \in i \mathbb{R}$ and $q \in Q_{n}$.

1.2. We now recall some ideas from [S]. For a group $G$, we will write $\widehat{G}$ for its unitary dual, and $\operatorname{Rep} G$ for the category of unitary representations of $G$. As before, let us write $G_{m}$ for $\mathrm{GL}(m, \mathbb{F})$. Let $P_{m}$ be the subgroup of matrices fixing the vector $(0, \ldots, 0,1)$

$$
P_{m} \approx G_{m-1} \ltimes \mathbb{F}^{m-1} .
$$

Now $G_{m-1}$ has exactly two orbits on $\left(\mathbb{F}^{m-1}\right)^{*} ; \quad 0$ and everything else. The stabilizer of 0 (the trivial character) is $G_{m-1}$ and that of a nontrivial character is isomorphic to $P_{m-1}$.

Thus, by Mackey theory, we have the functors $E: \widehat{G}_{m-1} \rightarrow \widehat{P}_{m}$ and $I: \widehat{P}_{m-1} \rightarrow \widehat{P}_{m}$ such that

$$
\widehat{P}_{m}=E\left(\widehat{G}_{m-1}\right) \amalg I\left(\widehat{P}_{m-1}\right) .
$$

Also, let $R$ be the restriction functor from $\operatorname{Rep} G_{m}$ to $\operatorname{Rep} P_{m}$. Then Kirillov's conjecture asserts

$$
R: \widehat{G}_{m} \rightarrow \widehat{P}_{m}
$$

Motivated by this conjecture, we call $\sigma$ in $\operatorname{Rep} G_{m}$ adducible of depth $k$ if

$$
R \sigma=I^{k-1} E(\tau)
$$

for some $\tau \in \operatorname{Rep} G_{m-k}$.

In this case we call $\tau$ the adduced representation and denote it by $A \sigma$.

The following result is an easy consequence of [S]. We include it only to motivate the subsequent results.

Proposition 0. For $s$ in $i \mathbb{R}$, the representations $R \sigma_{n}^{s}$ and $I E \sigma_{n-1}^{s}$ are isomorphic, irreducible, unitary representations of $P_{2 n}$.

The representation $R \sigma_{n}^{s}$ and $I E \sigma_{n-1}^{s}$ may be realized (naturally) upon fixed Hilbert spaces $H_{1}$ and $H_{2}$ (independent of $s$ ), and the proposition implies the existence of unitary operators $\left\{T_{s} \in \mathscr{U}\left(H_{1}, H_{2}\right)\right\}$ which intertwine the representations. The key observation in this paper is 
Lemma 1. There is a fixed unitary operator $T$ which intertwines the normalized representations $R \mathscr{N}_{n}^{s}$ and $I E \mathscr{N}_{n-1}^{s}$ for each $s$ in $i \mathbb{R}$.

This lemma will be proved in the next section. The main theorem is an easy consequence.

Theorem 1. (a) For each $g$ in $G_{2 n}$, the family $\left\{\mathscr{N}^{s}(g): s \in i \mathbb{R}\right\}$ may be extended to an analytic family $\left\{\mathscr{N}^{s}(g):|\operatorname{Re} s|<1 / 2\right\}$ of bounded operators on $L^{2}\left(M_{n}(\mathbb{F})\right)$.

(b) For a fixed $s$ in $\{|\operatorname{Re}(s)|<1 / 2\}$, the map $g \mapsto \mathscr{N}^{s}(g)$ gives a uniformly bounded representation of $G_{2 n}$ (the bound depends only on $n$ and $\left.|\operatorname{Re} s|\right)$. For $s$ in $(-1 / 2,1 / 2), \mathscr{N}^{s}$ is an irreducible unitary representation.

(c) For $s \in(-1 / 2,1 / 2), R \mathscr{N}_{n}^{s}$ is irreducible and isomorphic to $I E \mathscr{N}_{n-1}^{s}$.

\section{Proof of THE MaIN THEOREM}

The proofs are by direct computation. It may well be possible to give a more transparent, geometric argument, but I have not been able to find one.

In $\S \S 2.1-2.3, s$ is an imaginary number.

2.1. Partition $\mathbb{F}^{2 n}$ as $\mathbb{F}^{n-1} \oplus \mathbb{F} \oplus \mathbb{F}^{n-1} \oplus \mathbb{F}$ and accordingly decompose $2 n \times 2 n$ matrices into $4 \times 4$ block matrices. Then

$$
P=P_{2 n}=\left[\begin{array}{llll}
* & * & * & * \\
* & * & * & * \\
* & * & * & * \\
0 & 0 & 0 & 1
\end{array}\right]
$$

Let $\pi_{1}^{s}$ be the representation $\sigma^{s}$ restricted to $P$. Then by Mackey's subgroup theorem, $\pi_{1}^{s}$ is an induced representation, and may be explicitly realized on $H_{1}=L^{2}(M(n, \mathbb{F}))$ as follows.

Partition $\mathbb{F}^{n}$ as $\mathbb{F}^{n-1} \oplus \mathbb{F}$, and decompose $n \times n$ matrices as $2 \times 2$ block matrices. Let $F \in H_{1}$ and let

$$
p=\left[\begin{array}{llll}
a & d & g & j \\
b & e & h & k \\
c & f & i & \ell \\
0 & 0 & 0 & 1
\end{array}\right] \in P
$$

then

$$
\pi_{1}^{s}(p) F\left(\left[\begin{array}{ll}
x & z \\
y & t
\end{array}\right]\right)=\left|\left[\begin{array}{ll}
a^{\prime} & d^{\prime} \\
b^{\prime} & e^{\prime}
\end{array}\right]\right|^{-\left(\frac{n}{2}+s\right)}\left|i^{\prime}\right|^{\left(\frac{n}{2}+s\right)} F\left(\left[\begin{array}{ll}
x^{\prime} & z^{\prime} \\
y^{\prime} & t^{\prime}
\end{array}\right]\right)
$$

where

$$
\left[\begin{array}{llll}
1 & 0 & x & z \\
0 & 1 & y & t \\
0 & 0 & 1 & 0 \\
0 & 0 & 0 & 1
\end{array}\right]\left[\begin{array}{llll}
a & d & g & j \\
b & e & h & k \\
c & f & i & \ell \\
0 & 0 & 0 & 1
\end{array}\right]=\left[\begin{array}{llll}
a^{\prime} & d^{\prime} & 0 & 0 \\
b^{\prime} & e^{\prime} & 0 & 0 \\
c^{\prime} & f^{\prime} & i^{\prime} & \ell^{\prime} \\
0 & 0 & 0 & 1
\end{array}\right]\left[\begin{array}{llll}
1 & 0 & x^{\prime} & z^{\prime} \\
0 & 1 & y^{\prime} & t^{\prime} \\
0 & 0 & 1 & 0 \\
0 & 0 & 0 & 1
\end{array}\right]
$$


2.2. Imbed $G_{2 n-2}$ in $G_{2 n}$ as matrices of the form

$$
\left[\begin{array}{llll}
* & 0 & * & 0 \\
0 & 1 & 0 & 0 \\
* & 0 & * & 0 \\
0 & 0 & 0 & 1
\end{array}\right]
$$

Extend $\sigma_{n-1}^{s}$ trivially to

$$
P_{2 n-1}=\left[\begin{array}{cccc}
* & * & * & 0 \\
0 & 1 & 0 & 0 \\
* & * & * & 0 \\
0 & 0 & 0 & 1
\end{array}\right]
$$

and write $E \sigma_{n-1}^{s}$ for the extension.

Also, let us fix a nontrivial additive unitary character $\chi$ of $F$ as in [W] and use it to extend $E \sigma_{n-1}^{s}$ to $E \sigma_{n-1}^{s} \otimes \chi$ on

$$
P_{2 n-1} \ltimes \mathbb{F}^{2 n-1}=\left\{\left[\begin{array}{cccc}
* & * & * & * \\
0 & 1 & 0 & * \\
* & * & * & * \\
0 & 0 & 0 & 1
\end{array}\right]\right\}
$$

where the subgroup

$$
\left\{\left[\begin{array}{llll}
1 & 0 & 0 & j \\
0 & 1 & 0 & k \\
0 & 0 & 1 & \ell \\
0 & 0 & 0 & 1
\end{array}\right]\right\}
$$

acts by the character $\chi(k)$.

Let $\pi_{2}^{s}$ be the representation $I E \sigma_{n}^{s}=\operatorname{Ind}_{P_{2 n-1} \ltimes F^{2 n-1}}^{P}\left(E \sigma_{n}^{s} \otimes \chi\right)$. Then $\pi_{2}^{s}$ is realized on $L^{2}\left(\mathbb{F}^{2 n-1} ; L^{2}(M(n-1, \mathbb{F}))\right) \approx L^{2}\left(M(n-1, \mathbb{F}) \oplus \mathbb{F}^{2 n-1}\right) \equiv H_{2}$ as follows:

Decompose $\mathbb{F}^{2 n-1}$ as $\mathbb{F}^{n-1} \oplus \mathbb{F}^{n-1} \oplus \mathbb{F}$ and write $(y, \zeta, \tau)$ for a typical element in $\mathrm{F}^{2 n-1}$. Also let $x$ be a typical element in $M(n-1, \mathrm{~F})$. Then if $p \in P$ is as in (2), we have

(5) $\quad \pi_{2}^{s}(p) \varphi(x, y, \zeta, \tau)=|p|^{1 / 2} \chi\left(-k^{\prime \prime}\right)\left|a^{\prime \prime}\right|^{-\left(\frac{n-1}{2}+s\right)}\left|i^{\prime \prime}\right|^{\frac{n-1}{2}+s} \varphi\left(x^{\prime \prime}, y^{\prime \prime}, \zeta^{\prime \prime}, \tau^{\prime \prime}\right)$

where

$$
\left[\begin{array}{llll}
1 & 0 & 0 & 0 \\
\zeta & \tau & y & 0 \\
0 & 0 & 1 & 0 \\
0 & 0 & 0 & 1
\end{array}\right]\left[\begin{array}{llll}
a & d & g & j \\
b & e & h & k \\
c & f & i & \ell \\
0 & 0 & 0 & 1
\end{array}\right]=\left[\begin{array}{cccc}
\tilde{a} & \tilde{d} & \tilde{g} & \tilde{j} \\
0 & 1 & 0 & k^{\prime \prime} \\
\tilde{c} & \tilde{f} & \tilde{i} & \tilde{\ell} \\
0 & 0 & 0 & 1
\end{array}\right]\left[\begin{array}{cccc}
1 & 0 & 0 & 0 \\
\zeta^{\prime \prime} & \tau^{\prime \prime} & y^{\prime \prime} & 0 \\
0 & 0 & 1 & 0 \\
0 & 0 & 0 & 1
\end{array}\right]
$$

and

$$
\left[\begin{array}{ll}
1 & x \\
0 & 1
\end{array}\right]\left[\begin{array}{cc}
\tilde{a} & \tilde{g} \\
\tilde{c} & \tilde{i}
\end{array}\right]=\left[\begin{array}{cc}
a^{\prime \prime} & 0 \\
c^{\prime \prime} & i^{\prime \prime}
\end{array}\right]\left[\begin{array}{cc}
1 & x^{\prime \prime} \\
0 & 1
\end{array}\right]
$$


In the appendix it is shown that

$$
T_{s} \pi_{1}^{s}=\pi_{2}^{s} T_{s}
$$

with $T_{s}=C_{s}^{-1} \circ \mathscr{F}_{z t}$ where $\mathscr{F}_{z t}$ is the partial Fourier transform

$$
\left(\mathscr{F}_{z t} F\right)(x, y, \zeta, \tau)=\int \chi(\zeta z+\tau t) F\left(\left[\begin{array}{ll}
x & z \\
y & t
\end{array}\right]\right) d z d t
$$

and $C_{s}$ is the (unitary) change of variables

$$
C_{s} \varphi(x, y, \zeta, \tau)=|\tau|^{(n-1) / 2+s} \varphi(x, \zeta x+\tau y, \zeta, \tau) .
$$

2.3. Next we need to consider the normalized versions of $\pi_{1}^{s}$ and $\pi_{2}^{s}$. These are

$$
\tilde{\pi}_{1}^{s}=A_{1}(-s) \pi_{1}^{s} A_{1}(s)
$$

and

$$
\tilde{\pi}_{2}^{s}=A_{2}(-s) \pi_{2}^{s} A_{2}(s)
$$

where $A_{1}(s)$ and $A_{2}(s)$ are defined via the commutative diagrams

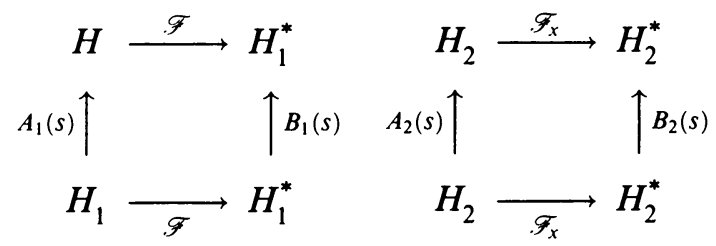

where $\mathscr{F}, \mathscr{F}_{x}$ are Fourier transforms

$$
\begin{gathered}
\mathscr{F} F\left(\left[\begin{array}{ll}
\xi & \eta \\
\zeta & \tau
\end{array}\right]\right)=\int \chi(\operatorname{tr}(\xi x+\eta y)+(\zeta z+\tau t)) F\left(\left[\begin{array}{ll}
x & z \\
y & t
\end{array}\right]\right) d x d y d z d t \\
\mathscr{F}_{x} \varphi(\xi, y, \zeta, \tau)=\int \chi(\operatorname{tr}(\xi x)) \varphi(x, y, \zeta, \tau) d x
\end{gathered}
$$

and $B_{1}(s), B_{2}(s)$ are the multiplication operators

$$
\begin{aligned}
B_{1}(s) \tilde{F}\left(\left[\begin{array}{ll}
\xi & \eta \\
\zeta & \tau
\end{array}\right]\right) & =\left|\left[\begin{array}{ll}
\xi & \eta \\
\zeta & \tau
\end{array}\right]\right|^{s} \widetilde{F}\left(\left[\begin{array}{ll}
\xi & \eta \\
\zeta & \tau
\end{array}\right]\right) \\
B_{2}(s) \tilde{\varphi}(\xi, y, \zeta, \tau) & =|\xi|^{s} \tilde{\varphi}(\xi, y, \zeta, \tau) .
\end{aligned}
$$

Now, using (8), (9) and (10) we see that

$$
A_{2}(-s) T_{s} A_{1}(s) \tilde{\pi}_{1}^{s}=\tilde{\pi}_{2}^{s} A_{2}(-s) T_{s} A_{1}(s) .
$$


The proof of Lemma 1 is now reduced to

Lemma 2. The operator $A_{2}(-s) T_{s} A_{1}(s)$ is independent of $s$.

Proof. Consider the following commutative diagram

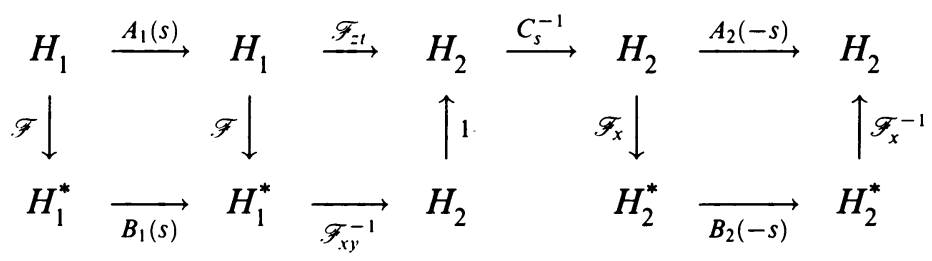

Now

$$
\begin{aligned}
& \left(C_{s} \mathscr{F}_{x}^{-1} B_{2}(s) \mathscr{F}_{y}^{-1}\right) \varphi(x, y, \zeta, \tau) \\
& =\left(\mathscr{F}_{x}^{-1} B_{2}(s) \mathscr{F}_{y}^{-1} \varphi\right)(x, \xi x+\tau y, \zeta, \tau)|\tau|^{(n-1) / 2+s} \\
& =\int \chi(-\operatorname{tr}(\xi x)) B_{2}(s) \mathscr{F}_{y}^{-1} \varphi(\xi, \zeta x+\tau y, \zeta, \tau)|\tau|^{(n-1) / 2+s} d \xi \\
& =\int \chi(-\operatorname{tr}(\xi x))|\xi|^{s}\left(\mathscr{F}_{y}^{-1} \varphi\right)(\xi, \zeta x+\tau y, \zeta, \tau)|\tau|^{(n-1) / 2+s} d \xi \\
& =\int \chi(-\operatorname{tr}(\xi x+\eta(\zeta x+\tau y)))|\xi|^{s} \varphi(\xi, \eta, \zeta, \tau)|\tau|^{(n-1) / 2+s} d \xi d \eta
\end{aligned}
$$

Let $\left(\begin{array}{ll}\xi^{\prime} & \eta^{\prime}\end{array}\right)=\left(\begin{array}{ll}\xi & \eta\end{array}\right)\left[\begin{array}{ll}1 & 0 \\ \zeta & \tau\end{array}\right]$. Then

$$
\begin{aligned}
& \text { (i) } d \xi^{\prime} d \eta^{\prime}=|\tau|^{(n-1) / 2} d \xi d \eta \\
& \text { (ii) }\left[\begin{array}{cc}
\xi^{\prime} & \eta^{\prime} \\
\zeta & \tau
\end{array}\right]=\left[\begin{array}{ll}
\xi & \eta \\
0 & 1
\end{array}\right]\left[\begin{array}{ll}
1 & 0 \\
\zeta & \tau
\end{array}\right] \Rightarrow\left|\left[\begin{array}{cc}
\xi^{\prime} & \eta^{\prime} \\
\zeta & \tau
\end{array}\right]\right|=|\xi||\tau| .
\end{aligned}
$$

So the integral becomes

$$
\int \chi\left(-\operatorname{tr}\left(\xi^{\prime} x+\eta^{\prime} y\right) D^{-1} \varphi\left(\xi^{\prime}, \eta^{\prime}, \zeta, \tau\right)\left|\begin{array}{cc}
\xi^{\prime} & \eta^{\prime} \\
\zeta & \tau
\end{array}\right|^{s} d \xi^{\prime} d \eta^{\prime}\right.
$$

where

$$
D \varphi(\xi, \eta, \zeta, \tau)=|\tau|^{(n-1) / 2} \varphi\left(\xi_{1}, \eta_{1}, \zeta, \tau\right)
$$

with

$$
\left(\begin{array}{ll}
\xi_{1} & \eta_{1}
\end{array}\right)=\left(\begin{array}{ll}
\xi & \eta
\end{array}\right)\left[\begin{array}{ll}
1 & 0 \\
\zeta & \tau
\end{array}\right] .
$$

But the last expression for the integral is

$$
\mathscr{F}_{x y}^{-1} B_{1}(s) D^{-1} \varphi(x, y, \zeta, \tau) .
$$

So we have

$$
\text { (11) } \mathscr{F}_{x y}^{-1} B_{1}(s) D^{-1}=C_{s} \mathscr{F}_{x}^{-1} B_{2}(s) \mathscr{F}_{y}^{-1} \Rightarrow B_{2}(-s) \mathscr{F}_{x} C_{s}^{-1} \mathscr{F}_{x y}^{-1} B_{1}(s)=\mathscr{F}_{y}^{-1} D \text {. }
$$

The operator $A_{2}(-s) T_{s} A_{1}(s)$ is given by the top row of the diagram. So (11) implies that it equals 


$$
\mathscr{F}_{x}^{-1}\left(\mathscr{F}_{y}^{-1} D\right) \mathscr{F}=\mathscr{F}_{x y}^{-1} D \mathscr{F}
$$

which is clearly independent of $s$. This proves Lemma 2 and also Lemma 1 .

2.4. Proof of Theorem 1. We proceed by induction on $n$. The case $n=1$ is classical (see $[\mathrm{K}]$ ). For the inductive step, we observe that the inductive hypothesis allows us to analytically continue $I E \mathscr{N}_{n-1}^{s}$ in the strip $\{|\operatorname{Re} s|<1 / 2\}$. Conjugating by $T$ we get the continuation of $\mathscr{N}_{n}^{s}$ restricted to $P=P_{2 n}$. But by (1) we have the (constant) analytic continuation on $Q$ and since $G_{2 n}$ is generated by $Q$ and $P$, this proves part (a).

Fix some element $w \in P_{2 n} \backslash Q_{n}$. Now there is a positive integer $K=K(n)$, such that every $g \in G_{2 n}$ is expressible as a word in the elements of $Q$, together with at most $K$ occurrences of $w$. By the inductive hypothesis $\mathscr{N}^{s}(w)$ is bounded if $|\operatorname{Re} s|<1$ (with the bound depending only on $|\operatorname{Re} s|$ ) and is unitary if $s$ is in $(-1 / 2,1 / 2)$. Since $\mathscr{N}^{s} \mid Q$ is unitary, this proves part (b).

Finally, part (c) is obvious.

Corollary. For $s$ in $(-1 / 2,1 / 2), A \sigma_{n}^{s}=\sigma_{n-1}^{s}$.

This is just a reformulation of part (c).

2.5. We give an application to $\operatorname{GL}(n, \mathbb{C})$. If $\nu$ is a unitary character of of $\mathbb{C}^{\times}$, let us write $\nu_{n}$ for the character of $\operatorname{GL}(n, \mathbb{C})$ given by $\nu_{n}(x)=\nu(\operatorname{det} x)$. Then it is easily seen that

$$
\begin{gathered}
A \nu_{n}=\nu_{n-1}, \\
A\left(\nu_{2 n} \otimes \sigma_{n}^{s}\right)=\nu_{2 n-2} \otimes \sigma_{n-1}^{s}, \quad \text { for } s \text { in }(-1 / 2,1 / 2) .
\end{gathered}
$$

Now, Vogan $[\mathrm{V}]$ has shown that every $\pi \in \widehat{\mathrm{GL}}(n, \mathbb{C})$ is of the form

$$
\pi=\pi_{1} \times \cdots \times \pi_{k}
$$

where $n=n_{1}+\cdots+n_{k}$, and each $\pi_{i} \in \widehat{\mathrm{GL}}\left(n_{i}, \mathbb{C}\right)$ is either a unitary character or a Stein representation tensored with a unitary character. (The right side of (15) is Zelevinsky's notation for $\operatorname{Ind}_{P_{n_{1} \ldots . n_{k}}}^{G_{n}}\left(\pi_{1} \otimes \cdots \otimes \pi_{k}\right)$.)

On the other hand in $[\mathrm{S}]$ we showed that

$$
A\left(\pi_{1} \times \cdots \times \pi_{k}\right)=A \pi_{1} \times \cdots \times A \pi_{k} .
$$

Now (13), (14) allow one to compute the right side of (16). Thus we get a description of $A \pi$ in terms of Vogan's classification.

\section{APPENDIX}

We sketch the proof of the intertwining relation (8) asserted in $\S 2.2$.

First observe that (3) may be rewritten as

$$
\pi_{1}^{s}(p) F\left(\left[\begin{array}{cc}
x & z \\
y & t
\end{array}\right]\right)=|p|^{-\frac{n}{2}-s}\left|i^{\prime}\right|^{n+2 s} F\left(\left[\begin{array}{cc}
x^{\prime} & z^{\prime} \\
y^{\prime} & t^{\prime}
\end{array}\right]\right)
$$


and that $i^{\prime}$ depends only on $p$ and $(x, y)$. In fact, if (4) is broken up into

$$
\left[\begin{array}{llll}
1 & 0 & x & 0 \\
0 & 1 & y & 0 \\
0 & 0 & 1 & 0 \\
0 & 0 & 0 & 1
\end{array}\right]\left[\begin{array}{llll}
a & d & g & j \\
b & e & h & k \\
c & f & i & \ell \\
0 & 0 & 0 & 1
\end{array}\right]=\left[\begin{array}{cccc}
\bar{a} & \bar{d} & 0 & \bar{j} \\
\bar{b} & \bar{e} & 0 & \bar{k} \\
\bar{c} & \bar{f} & \bar{i} & \bar{\ell} \\
0 & 0 & 0 & 1
\end{array}\right]\left[\begin{array}{llll}
1 & 0 & x^{\prime} & 0 \\
0 & 1 & y^{\prime} & 0 \\
0 & 0 & 1 & 0 \\
0 & 0 & 0 & 1
\end{array}\right]
$$

and

$$
\left[\begin{array}{llll}
1 & 0 & 0 & z \\
0 & 1 & 0 & t \\
0 & 0 & 1 & 0 \\
0 & 0 & 0 & 1
\end{array}\right]\left[\begin{array}{cccc}
\bar{a} & \bar{d} & 0 & \bar{j} \\
\bar{b} & \bar{e} & 0 & \bar{k} \\
\bar{c} & \bar{f} & \bar{i} & \bar{\ell} \\
0 & 0 & 0 & 1
\end{array}\right]=\left[\begin{array}{llll}
a^{\prime} & d^{\prime} & 0 & 0 \\
b^{\prime} & e^{\prime} & 0 & 0 \\
c^{\prime} & f^{\prime} & i^{\prime} & \ell^{\prime} \\
0 & 0 & 0 & 1
\end{array}\right]\left[\begin{array}{llll}
1 & 0 & 0 & z^{\prime} \\
0 & 1 & 0 & t^{\prime} \\
0 & 0 & 1 & 0 \\
0 & 0 & 0 & 1
\end{array}\right]
$$

then

$$
i^{\prime}=\bar{i}
$$

Next, observe that (6) and (7) may be combined as

$$
\left[\begin{array}{llll}
1 & 0 & x & 0 \\
\zeta & \tau & y & 0 \\
0 & 0 & 1 & 0 \\
0 & 0 & 0 & 1
\end{array}\right]\left[\begin{array}{llll}
a & d & g & j \\
b & e & h & k \\
c & f & i & \ell \\
0 & 0 & 0 & 1
\end{array}\right]=\left[\begin{array}{cccc}
a^{\prime \prime} & d^{\prime \prime} & 0 & j^{\prime \prime} \\
0 & 1 & 0 & k^{\prime \prime} \\
c^{\prime \prime} & f^{\prime \prime} & i^{\prime \prime} & \ell^{\prime \prime} \\
0 & 0 & 0 & 1
\end{array}\right]\left[\begin{array}{cccc}
1 & 0 & x^{\prime \prime} & 0 \\
\zeta^{\prime \prime} & \tau^{\prime \prime} & y^{\prime \prime} & 0 \\
0 & 0 & 1 & 0 \\
0 & 0 & 0 & 1
\end{array}\right]
$$

and (5) becomes

(22)

$$
\pi_{2}^{s}(p) \varphi(x, y, \zeta, \tau)=|p|^{1-\frac{n}{2}-s} \chi\left(-k^{\prime \prime}\right)\left(|\tau|^{-1}\left|\tau^{\prime \prime}\right|\left|i^{\prime \prime}\right|^{2}\right)^{\frac{n-1}{2}+s} \varphi\left(x^{\prime \prime}, y^{\prime \prime}, \zeta^{\prime \prime}, \tau^{\prime \prime}\right) .
$$

Now

$$
\left[\begin{array}{llll}
1 & 0 & 0 & 0 \\
\zeta & \tau & 0 & 0 \\
0 & 0 & 1 & 0 \\
0 & 0 & 0 & 1
\end{array}\right]\left[\begin{array}{llll}
1 & 0 & x & 0 \\
0 & 1 & y & 0 \\
0 & 0 & 1 & 0 \\
0 & 0 & 0 & 1
\end{array}\right]=\left[\begin{array}{cccc}
1 & 0 & x & 0 \\
\zeta & \tau & \zeta x+\tau y & 0 \\
0 & 0 & 1 & 0 \\
0 & 0 & 0 & 1
\end{array}\right]
$$

So if $C_{s}$ is the (unitary) operator

$$
C_{s} \varphi(x, y, \zeta, \tau)=|\tau|^{(n-1) / 2+s} \varphi(x, \zeta x+\tau y, \zeta, \tau),
$$

then

$$
C_{s} \pi_{2}^{s}(p) C_{s}^{-1} \varphi(x, y, \zeta, \tau)=|p|^{1-\frac{n}{2}-s} \chi\left(-k^{\prime}\right)\left|i^{\prime}\right|^{n-1+2 s} \varphi\left(x^{\prime}, y^{\prime}, \zeta^{\prime}, \tau^{\prime}\right)
$$

where $x^{\prime}, y^{\prime}$ are determined by (18), and $i^{\prime}, k^{\prime}, \zeta^{\prime}, \tau^{\prime}$ are determined by

$$
\left[\begin{array}{llll}
1 & 0 & 0 & 0 \\
\zeta & \tau & 0 & 0 \\
0 & 0 & 1 & 0 \\
0 & 0 & 0 & 1
\end{array}\right]\left[\begin{array}{cccc}
\bar{a} & \bar{d} & 0 & \bar{j} \\
\bar{b} & \bar{e} & 0 & \bar{k} \\
\bar{c} & \bar{f} & \bar{i} & \bar{\ell} \\
0 & 0 & 0 & 1
\end{array}\right]=\left[\begin{array}{cccc}
* & * & 0 & * \\
0 & 1 & 0 & k^{\prime} \\
* & * & i^{\prime} & * \\
0 & 0 & 0 & 1
\end{array}\right]\left[\begin{array}{cccc}
1 & 0 & 0 & 0 \\
\zeta^{\prime} & \tau^{\prime} & 0 & 0 \\
0 & 0 & 1 & 0 \\
0 & 0 & 0 & 1
\end{array}\right] .
$$

From (25) it is clear that $i^{\prime}=\bar{i}$ as before, and that

$$
k^{\prime}=\zeta \bar{j}+\tau \bar{k}
$$


and

$$
\left(\begin{array}{ll}
\zeta^{\prime} & \tau^{\prime}
\end{array}\right)=\left(\begin{array}{ll}
\zeta & \tau
\end{array}\right)\left[\begin{array}{ll}
\bar{a} & \bar{d} \\
\bar{b} & \bar{e}
\end{array}\right] .
$$

Taking the Fourier transform of (17) we get

(28) $\mathscr{F}_{z t} \pi_{1}^{s}(p) F(x, y, \zeta, \tau)=\int \chi(\zeta z+\tau t)|p|^{-\frac{n}{2}-s}\left|i^{\prime}\right|^{n+2 s} F\left(\left[\begin{array}{ll}x^{\prime} & z^{\prime} \\ y^{\prime} & t^{\prime}\end{array}\right]\right) d z d t$

Now from (19) it follows that

$$
\left[\begin{array}{l}
z \\
t
\end{array}\right]+\left[\begin{array}{l}
\bar{j} \\
\bar{k}
\end{array}\right]=\left[\begin{array}{ll}
a^{\prime} & d^{\prime} \\
b^{\prime} & e^{\prime}
\end{array}\right]\left[\begin{array}{l}
z^{\prime} \\
t^{\prime}
\end{array}\right]
$$

and that

$$
\left[\begin{array}{ll}
a^{\prime} & d^{\prime} \\
b^{\prime} & e^{\prime}
\end{array}\right]=\left[\begin{array}{ll}
\bar{a} & \bar{d} \\
\bar{b} & \bar{e}
\end{array}\right]
$$

and thus

$$
\left[\begin{array}{l}
z \\
t
\end{array}\right]=\left[\begin{array}{ll}
\bar{a} & \bar{d} \\
\bar{b} & \bar{e}
\end{array}\right]\left[\begin{array}{l}
z^{\prime} \\
t^{\prime}
\end{array}\right]-\left[\begin{array}{l}
\bar{j} \\
\bar{k}
\end{array}\right]
$$

Also, from (18) and (20) we have

$$
\left|\begin{array}{ll}
\bar{a} & \bar{d} \\
\bar{b} & \bar{e}
\end{array}\right|=|p||\bar{i}|^{-1}=|p|\left|i^{\prime}\right|^{-1} .
$$

Making the change of variables (29) in (28) and using (26), (27) and (30), we get

$$
|p|^{1-\frac{n}{2}-s} \chi\left(-k^{\prime}\right)\left|i^{\prime}\right|^{n-1+2 s} \mathscr{F}_{z t} \varphi\left(x^{\prime}, y^{\prime}, \zeta^{\prime}, \tau^{\prime}\right)
$$

And so $\mathscr{F}_{z t} \pi_{1}^{s}(p) \mathscr{F}_{z t}^{-1}=C_{s} \pi_{2}^{s}(p) C_{s}^{-1}$ which gives (8).

\section{REFERENCES}

[B] J. Bernstein, P-invariant distributions on $\mathrm{GL}(n)$ and the classification of unitary representation of $\mathrm{GL}(n)$ (non-Archimedean case), in "Lie Group Representations II", Proceedings, University of Maryland 1982-83, (R . Herb et al., eds.) SLNM 1041.

[K] R. Kunze and E. Stein, Uniformly bounded representations and harmonic analysis of the $2 \times 2$ real unimodular group, Amer. J. Math. 82 (1960), 1-62.

[S] S. Sahi, On Kirillov's conjecture for archimedean fields, Compos. Math. (to appear).

[St] E. Stein, Analysis in matrix space and some new representations of $S L(n, \mathbb{C})$, Ann. of Math. 86 (1967), 461-490.

[V] D. Vogan, The unitary dual of $G L(n)$ over an archimedean field, Invent. Math. 83 (1986), 449-505.

[W] A. Weil, Basic number theory, Springer-Verlag, New York.

Department of Mathematics, Princeton University, Princeton New Jersey 08544

Mathematical Sciences Research Institute, Berkeley, California 94720 